Service social

\title{
Les stratégies pour favoriser l'engagement au sein des groupes visant à contrer la violence conjugale
}

\section{Valérie Roy, Michel Labarre et Marie-Ève Drouin}

Volume 61, numéro 1, 2015

Être reconnu et protégé

URI : https://id.erudit.org/iderudit/1033741ar

DOI : https://doi.org/10.7202/1033741ar

Aller au sommaire du numéro

\section{Éditeur(s)}

École de service social de l’Université Laval

ISSN

1708-1734 (numérique)

Découvrir la revue

Citer cet article

Roy, V., Labarre, M. \& Drouin, M.-È. (2015). Les stratégies pour favoriser l'engagement au sein des groupes visant à contrer la violence conjugale. Service social, 61(1), 77-92. https://doi.org/10.7202/1033741ar
Résumé de l'article

$\mathrm{Au}$ Québec et ailleurs, les groupes pour les conjoints ayant des comportements violents constituent une des réponses sociales au problème de la violence conjugale. Or, les taux d'abandon dans ces groupes demeurent préoccupants en raison notamment des risques de récidive qui y sont associés. Les études suggèrent qu'un faible sentiment d'engagement compte parmi les facteurs associés aux abandons, alors que l'engagement dans les groupes serait considéré comme un élément favorisant le succès des programmes d'intervention. Cet article présente les résultats d'une recherche qualitative sur les stratégies qui peuvent favoriser l'engagement des hommes dans les programmes de groupe. Les résultats mettent en lumière l'importance du choix de s'engager dans le groupe, de même que le rôle des intervenants, des autres membres du groupe et de l'environnement dans ce processus. Une analyse critique des stratégies contribuant à maintenir et à renouveler le choix des hommes de s'engager dans leur démarche de groupe est proposée. 


\title{
Les stratégies pour favoriser l'engagement au sein des groupes visant à contrer la violence conjugale
}

\author{
ROY, Valérie Roy ${ }^{1}$ \\ LABARRE, Miche1 2 \\ DROUIN, Marie-Ève 3
}

\begin{abstract}
RÉSUMÉ
$\mathrm{Au}$ Québec et ailleurs, les groupes pour les conjoints ayant des comportements violents constituent une des réponses sociales au problème de la violence conjugale. Or, les taux d'abandon dans ces groupes demeurent préoccupants en raison notamment des risques de récidive qui y sont associés. Les études suggèrent qu'un faible sentiment d'engagement compte parmi les facteurs associés aux abandons, alors que l'engagement dans les groupes serait considéré comme un élément favorisant le succès des programmes d'intervention. Cet article présente les résultats d'une recherche qualitative sur les stratégies qui peuvent favoriser l'engagement des hommes dans les programmes de groupe. Les résultats mettent en lumière l'importance du choix de s'engager dans le groupe, de même que le rôle des intervenants, des autres membres du groupe et de l'environnement dans ce processus. Une analyse critique des stratégies contribuant à maintenir et à renouveler le choix des hommes de s'engager dans leur démarche de groupe est proposée.
\end{abstract}

Mots clés : engagement, intervention de groupe, violence conjugale, hommes, abandon.

\begin{abstract}
In Quebec and elsewhere, group work programs for violent partners are widely used in order to address the social problem of intimate partner violence. Dropout rates in these groups however remain of concerns because of the associated risks of recidivism. Studies suggest that a low sense of engagement in these groups is a dropout factor, while group engagement is considered as an element contributing to programs success. This article presents the results of a qualitative research on the strategies that may foster men's engagement in their group program. Results highlight the importance of men's choice to commit themselves to the group, along with practitioners, group members and environment roles in the engagement process. Discussion offers a critical analysis of the strategies that may foster and support the choice of men to engage in their group program.
\end{abstract}

Keywords: engagement, group work, intimate partner violence, men, dropout.

1. Valérie Roy, Ph.D., T.S., est professeure agrégée à l'École de service social de l'Université Laval. Ses activités de recherche et d'enseignement concernent principalement le service social des groupes et la violence conjugale.

2. Michel Labarre est étudiant au doctorat en service social à l'Université Laval. Ses intérêts de recherche portent principalement sur la paternité et la violence conjugale.

3. Marie-Ėve Drouin est professionnelle de recherche au Centre de recherche sur l'adaptation des jeunes et des familles à risque (JEFAR). 


\section{INTRODUCTION}

Par ses nombreuses conséquences, la violence conjugale reste un problème social d'importance partout dans le monde (Organisation mondiale de la Santé, 2014). Au Canada, en 2013, plus de 90300 personnes, dont près de $80 \%$ sont des femmes, ont été victimes de violence de la part de leur partenaire (Statistique Canada, 2015). Au Québec, pour la même année, ce sont 18885 personnes qui en ont été victimes et, près de huit fois sur dix, l'auteur présumé était un homme (Ministère de la Sécurité publique, 2015). Ce chiffre ne comprend de surcroît que les incidents rapportés à la police et ne représente donc qu'une fraction du nombre réel de victimes.

Une des réponses sociales à ce problème consiste à offrir une aide psychosociale aux conjoints qui ont des comportements violents (Gouvernement du Québec, 1995), qui prend le plus souvent la forme de programmes de groupe thérapeutique ou éducatif. Or, les études rapportent que les hommes persévèrent peu dans ces programmes (Brodeur, Rondeau, Brochu, Lindsay et Phelps, 2008 ; Holdsworth, Bowen, Brown et Howat, 2014 ; Jewell et Wormith, 2010). Jewell et Wormith (2010) indiquent que, selon les études, entre $22 \%$ et $78 \%$ des hommes ayant entrepris un programme ne le terminent pas. Ces abandons sont préoccupants puisqu'ils augmentent les risques de récidive (Bennett, Stoops, Call et Flett, 2007).

Un des facteurs qui expliquent ces nombreux abandons est le faible sentiment d'engagement de certains hommes dans la démarche de groupe (Chovanec, 2014 ; Rondeau, Brochu, Lemire et Brodeur, 1999). Bien qu'il n'y ait pas une définition qui fasse consensus, l'engagement est le plus souvent défini comme un concept multidimensionnel qui traduit l'implication du client dans la démarche d'intervention (Holdsworth et al., 2014 ; Macgowan, 2006). Les dimensions utilisées pour évaluer cette implication varient grandement selon les auteurs (Holdsworth et al., 2014 ; Semiatin, Murphy et Elliott, 2013 ; Ting, Jordan-Green, Murphy et Pitts, 2009). Le modèle de Macgowan (2006) intègre plusieurs de ces dimensions, soit des dimensions relatives à l'individu (présence, contribution aux échanges, travail sur ses problèmes, respect du contrat de groupe), aux intervenants (relation avec les intervenants) et au groupe (relation avec les membres, aider les autres). Un homme engagé est donc celui qui s'investit minimalement dans chacune de ces dimensions et cet engagement est considéré comme un gage de succès des programmes d'intervention en matière de violence conjugale (Contrino, Dermen, Nochajski, Wieczorek et Navratil, 2007 ; Holdsworth et al., 2014).

Dès lors, des recherches se sont intéressées aux stratégies susceptibles de stimuler l'engagement des hommes dans les programmes de groupe. Certaines sont associées à la motivation des hommes, aux caractéristiques des programmes ou encore à la relation avec l'intervenant. Parmi les stratégies centrées sur la motivation des hommes, certaines peuvent être utilisées avant l'intégration au groupe, par exemple des entretiens motivationnels afin d'augmenter la volonté de ces derniers à entreprendre une démarche de changement (Crane et Eckhardt, 2013 ; Murphy, Linehan, Reyner, Musser et Taft, 2012 ; Scott, King, McGinn et Hosseini, 2011 ; Zalmanowitz, Babins-Wagner, Rodger, Corbett et Leschied, 2013). Les études suggèrent que cette stratégie favorise la persévérance dans le programme. Elle n'a toutefois pas été associée à un engagement plus solide au groupe ou à de plus faibles taux de récidive (Crane et Eckhardt, 2013 ; Murphy et al., 2012 ; Scott et al., 2011). 
Dans certains programmes, des activités (ou devoirs) entre les rencontres sont prévues pour aider les hommes à maintenir leurs efforts de changement entre les rencontres de groupe. Pour Holdsworth et al. (2014), l'assiduité dans la réalisation de ces activités favorise l'intégration et l'application du contenu du programme. La réalisation de ces activités est d'ailleurs associée à l'engagement des hommes envers le programme (Ting et al., 2009), ainsi qu'aux comportements pro-thérapeutiques à la fin du groupe (Semiatin et al., 2013).

Dans l'organisation des activités au sein du groupe, on suggère des stratégies permettant aux hommes de raconter leurs expériences afin d'augmenter l'engagement dans le processus d'intervention (Chovanec, 2014). Ces stratégies visent à renforcer les relations entre les membres du groupe pour soutenir leurs efforts de changement (Chovanec, 2012 ; 2014). II importe de demeurer vigilant afin de s'assurer que le renforcement des liens entre les membres n'ait pas un effet de collusion, comme l'ont constaté Sullivan et Claes (2015). Les stratégies sur les activités au sein du groupe demeurent d'ailleurs à documenter (Holdsworth et al., 2014).

Plusieurs recherches ont aussi examiné l'influence de l'intervenant, particulièrement sous l'angle du lien de confiance (Campbell, Neil, Jaffe et Kelly, 2010 ; Holdsworth et al., 2014). À l'importance de la création de ce lien s'ajoutent, au registre des stratégies susceptibles de favoriser l'engagement dans les groupes, l'attitude non directive de l'intervenant, le soutien, la confrontation et le renforcement des progrès réalisés (Holdsworth et al., 2014). L'intervenant peut aussi vérifier si des obstacles environnementaux ( $p$. ex. conflits d'horaire) peuvent être résolus (Chovanec, 2014). Or, à l'instar des stratégies centrées sur le programme, les stratégies s'adressant aux facteurs externes qui peuvent influencer l'engagement sont moins connues que celles centrées sur le client et l'intervenant (Holdsworth et al., 2014).

Les connaissances sur les stratégies visant à favoriser l'engagement dans les groupes suggèrent donc la pertinence d'agir sur un ensemble de facteurs, liés aux caractéristiques des hommes (p. ex. motivation), des programmes (p. ex. activités), de l'intervenant (p. ex. attitudes), ainsi qu'aux facteurs externes qui restent à explorer. Afin de mieux comprendre ces facteurs et leur interaction, une recherche sur le processus d'engagement des hommes a été réalisée. Cette recherche poursuivait trois objectifs: (1) identifier les perceptions que les hommes ont de l'engagement dans la démarche de groupe ; (2) connaître leurs points de vue sur les éléments qui le favorisent et lui font obstacle ; et 3) identifier les stratégies qui favorisent l'engagement dans la démarche. Cet article s'intéresse aux résultats liés aux stratégies favorisant l'engagement.

\section{CADRe THÉorique}

Considérant que le but de la recherche est d'avoir une compréhension holistique du processus d'engagement, le modèle écologique de Bronfenbrenner (1986) a été retenu. Selon ce modèle, l'humain évolue dans un environnement composé de six systèmes interdépendants. L'ontosystème réfère aux caractéristiques individuelles ( $p$. ex. âge, niveau d'étude). Le microsystème est l'environnement immédiat dans lequel l'individu évolue : dans le cadre de la recherche, le groupe, incluant ses dimensions structurelles et relationnelles, constitue le principal microsystème étudié. L'individu évolue aussi au sein d'autres microsystèmes ( $p$. ex. famille, travail) et les interactions entre ces microsystèmes et le groupe constituent le mésosystème. 
L'exosystème correspond aux organisations qui influent sur la vie de l'individu sans que celui-ci y soit directement impliqué (p. ex. les politiques de l'organisme qui dispense le groupe), alors que le macrosystème renvoie aux croyances et valeurs qui coexistent au sein d'une société ( $p$. ex. représentation sociale de la violence conjugale). Le chronosystème réfère au passage du temps et a été intégré à l'ontosystème ( $p$. ex. événements marquants de la vie des participants) et au microsystème (avancement dans la démarche de groupe).

\section{MÉTHODOLOGIE}

Étant donné l'objectif de l'étude, une approche qualitative a été privilégiée afin d'avoir accès aux points de vue des hommes sur le processus d'engagement dans les groupes visant à contrer la violence conjugale. Ce type d'approche est d'ailleurs suggéré pour étudier le processus thérapeutique des hommes qui participent à ces groupes, aspect souvent négligé dans les études sur les programmes en violence conjugale (Bowen, 2011).

\section{Participants}

Les participants ont été recrutés en deux étapes distinctes et consécutives, selon la logique d'un échantillon intentionnel d'hommes ayant l'expérience d'un groupe de thérapie en violence conjugale (Patton, 2002). Lors de la première étape, 27 participants ont été recrutés pour participer à une entrevue individuelle. Tous étaient des francophones nés au Québec. L'âge moyen était de 36 ans (ET=10.8). Parmi eux, 14 (52\%) avaient un diplôme d'études de niveau secondaire ou supérieur ; 12 (45\%) étaient en relation avec une conjointe, et 18 (66\%) assumaient un rôle parental auprès d'au moins un enfant. Neuf hommes avaient un revenu annuel de moins de 20000 \$, neuf entre 20000 \$ et 40000 \$ et neuf bénéficiaient d'un revenu supérieur à $40000 \$$. Huit avaient une obligation judiciaire de participer au programme. Lors de la seconde étape, 13 hommes, n'ayant pas participé aux entrevues, ont été recrutés pour former deux groupes de discussion. Ils étaient aussi tous des francophones nés au Québec, mais ils étaient un peu plus âgés (âge moyen de 44 ans) et bénéficiaient dans une plus grande proportion (46\%) de revenus supérieurs à 40000 \$. Leur situation conjugale était relativement semblable (54\% en couple), mais ils étaient plus nombreux à exercer un rôle parental (12 sur 13). Cinq hommes avaient une obligation judiciaire de participer au groupe.

\section{Échantillon et contexte}

Les participants ont été recrutés dans deux organismes, situés dans deux régions du Québec, offrant des groupes de thérapie visant à contrer la violence conjugale. Ces groupes sont composés de huit hommes et sont animés par deux intervenants ayant une formation dans une discipline des sciences sociales. Les hommes qui prennent part à ces groupes doivent compléter un minimum de 20 rencontres d'environ $2 \mathrm{~h} 30$ chacune, à raison d'une rencontre par semaine. Généralement, deux entrevues d'évaluation précèdent le groupe. Les groupes sont semi-ouverts, c'est-à-dire qu'ils incluent des hommes à différentes étapes de leur démarche.

Afin d'obtenir une diversité dans les expériences des participants (Patton, 2002), les hommes ont été recrutés avec le souci d'inclure différentes expériences en termes de persévérance au groupe et de nombres de rencontres effectuées. Ainsi, pour les hommes ayant pris part aux 
entrevues individuelles, 9 étaient au début de leur démarche (de 4 à 10 rencontres), 15 étaient à la fin de leur démarche (de 11 à 25 rencontres), et 3 avaient abandonné le groupe. Chez les hommes ayant participé aux groupes de discussion (un groupe dans chaque organisme), quatre étaient en début de processus et neuf étaient en fin de démarche.

\section{Procédures de recrutement}

Lors de leur troisième rencontre de groupe, chaque homme était informé de la recherche par les intervenants de son groupe. Les hommes intéressés signaient alors un premier formulaire de consentement pour accepter qu'un membre de l'équipe communique avec eux pour leur donner plus d'information et planifier un moment pour réaliser l'entrevue. Avant le début de l'entrevue, les hommes ont signé un second consentement à participer à l'étude. La même procédure a été utilisée pour les groupes de discussion, suivant les règles éthiques en vigueur à l'Université Laval.

\section{Entrevues et groupes de discussion}

Les entrevues, de type semi-structuré, ont été réalisées par deux assistants de recherche. Elles comportaient trois thèmes principaux, inspirés du modèle écologique et du guide d'entrevue de Chovanec (2012). Le premier thème portait sur la perception de l'engagement et le second traitait des facteurs pouvant influer sur l'engagement. Le dernier thème portait sur les stratégies et était constitué de trois mises en situation (vignettes) pour lesquelles les participants devaient identifier des moyens susceptibles de favoriser l'engagement des hommes. L'utilisation de vignettes, faisant ressortir les préoccupations des organismes partenaires et des facteurs associés à l'abandon, a été retenue comme stratégie afin d'enrichir la collecte de données.

La première décrivait la situation de Pierre qui, ayant complété 12 rencontres de groupe, pense à abandonner le programme en raison de la diminution de ses comportements violents et du temps qu'il pourrait consacrer à son travail et à ses enfants. Dans la seconde, Jean pense à abandonner le groupe en raison du dénigrement de ses amis, du manque de soutien qu'il perçoit chez sa conjointe et de conflits d'horaire avec son emploi. Dans la dernière, Jacques, plutôt timide, songe à abandonner le groupe puisqu'il y a été la cible de moqueries et qu'il se reconnaît peu dans les situations des autres membres, dont certains par ailleurs monopolisent les discussions. Au cours des entrevues, plusieurs participants ont fait des liens entre les trois vignettes et leur situation personnelle ou celles vécues par des membres de leur groupe. Deux entrevues prétests ont permis d'assurer la clarté et la pertinence des questions.

Afin d'assurer la crédibilité de la recherche, deux groupes de discussion ont eu lieu à la suite de l'analyse des données des entrevues (Patton, 2002). Lors de ces groupes, les résultats préliminaires ont été présentés aux participants, qui ont donné leurs points de vue à propos des principaux résultats sur la base de leurs propres expériences. Ces groupes ont permis d'observer une certaine saturation des données puisque leur contenu appuyait essentiellement les résultats en exemplifiant davantage certaines stratégies identifiées par le biais des entrevues individuelles. Les données recueillies lors des groupes de discussion ont été intégrées au corpus des données. 


\section{Analyse des données}

Les entrevues et les groupes de discussion ont été enregistrés numériquement, transcrits intégralement, pour ensuite faire l'objet d'une analyse de contenu (Miles et Huberman, 2003) par l'équipe de recherche. De façon plus spécifique aux stratégies favorisant l'engagement des hommes, suivant un processus d'analyse inductive, les points de vue des 27 participants sur les vignettes ont fait l'objet d'une première codification libre par une professionnelle de recherche. La catégorisation ainsi obtenue a ensuite été discutée, synthétisée et hiérarchisée avec la chercheure principale. Par la suite, l'ensemble des entrevues et des groupes de discussion a entièrement été relu par un étudiant de doctorat, en collaboration avec la professionnelle de recherche et la chercheure, pour identifier d'autres stratégies susceptibles de bonifier l'arborescence élaborée à partir des vignettes. L'analyse des entrevues et des groupes de discussion a permis d'identifier 60 moyens ou éléments concrets, qui ont été regroupés sous huit stratégies plus générales favorisant l'engagement dans les groupes. Ces stratégies ont ensuite été associées aux différents systèmes du modèle écologique de Bronfenbrenner (1986).

\section{RÉSULTATS}

Deux stratégies sont associées aux hommes (ontosystème), alors qu'une stratégie concerne les intervenants, les membres et le fonctionnement du groupe (microsystème). Deux stratégies ont trait aux interactions entre le groupe et les autres sphères de vie des hommes (mésosystème), deux autres aux politiques des organismes (exosystème) et une dernière stratégie au contexte social (macrosystème).

\section{Stratégies associées aux hommes}

Les propos des participants ont permis d'identifier deux stratégies qui relèvent des hommes qui effectuent une démarche pour contrer leurs comportements violents : choisir de s'engager et prendre le risque de s'investir dans la démarche.

\section{Choisir de s'engager}

Selon les propos des hommes rencontrés, la notion de choix est cruciale pour l'engagement dans les groupes. Ce choix s'appuie sur un ensemble d'éléments, notamment les motivations à effectuer une démarche de groupe, et s'effectue au terme d'une évaluation des coûts et avantages associés à l'engagement.

Les propos des participants illustrent la diversité des motivations à effectuer une démarche de groupe en violence conjugale. Pour certains, l'engagement relève davantage d'une motivation extrinsèque, liée aux gains externes susceptibles de résulter de la participation au groupe, comme récupérer la garde de ses enfants ou éviter la prison :

Ce n'est pas compliqué, j'ai 35 ans pis je suis rendu où je voulais dans la vie. J'ai ma maison, j'ai mes affaires. Pis je n'ai pas le goût de recommencer. (...) Si je ne suis pas ça [le groupe], je m'en vais en-dedans [pour] longtemps, fait que je perds tout. Fait que je me dis "six mois de thérapie, il y a moyen de s'arranger". (...) J'en ai fait plein de prison pis à chaque fois, tu sors avec ton petit sac pis tu 
recommences. Là, ça ne me tente pas, j'ai comme pas mal à perdre. (participant 21, 8 rencontres).

Pour d'autres, la motivation à s'engager dans le groupe est davantage intrinsèque, donc liée à un désir de changement personnel. Pour eux, la décision d'effectuer une démarche est souvent précédée d'une prise de conscience ou de l'atteinte d'un point de rupture (avoir peur de soi, atteindre un seuil de mal-être intolérable, prendre conscience de la gravité de la situation) et s'accompagne de gains potentiels sur le plan personnel :

Je n'ai jamais levé la main sur mes enfants. Ça a toujours été de la violence psychologique et verbale. Un jour, j'ai voulu me rapprocher de mon enfant pour le caresser. Et là, il s'est comme vraiment tassé pour m'éviter comme si j'étais pour le frapper. À ce moment-là, je me suis dit "Wow ! Ça va loin. Ça va trop loin pis faut que je fasse de quoi." (participant 2, 24 rencontres)

Les propos des participants suggèrent également que les motivations à participer au groupe peuvent évoluer en cours de démarche. Le choix de s'engager peut donc s'effectuer indépendamment de la nature des motivations initiales à effectuer une démarche. Plusieurs hommes ont d'ailleurs mentionné qu'ils ont débuté un groupe en raison de facteurs externes ( $p$. ex. contrainte judiciaire) et qu'en cours de démarche, ils ont choisi de tenter d'en tirer des avantages pour eux-mêmes: «Je me suis dit: écoute, tu n'as pas le choix d'aller suivre 20 séances, (...) Je vais me retrousser les manches pis je vais essayer d'attraper quelque chose de positif à travers ça. Je n'irai pas perdre mon temps. » (participant 17, 7 rencontres)

Les propos des hommes rencontrés suggèrent que ces motivations initiales, intrinsèques ou extrinsèques, favorisent la présence au groupe. Par contre, pour que cette présence se transforme en engagement, il est nécessaire d'effectuer un choix personnel d'engagement dans le groupe. Plusieurs hommes insistent d'ailleurs sur l'importance de choisir d'effectuer une démarche pour soi et non pour les autres : « S'engager dans un groupe c'est décider de mettre du temps pour prendre du temps pour soi, prendre du temps pour se soigner (...) vraiment décider de régler un problème » (participant 2, 24 rencontres). Ainsi, bien que les motivations initiales puissent être extrinsèques, il semble nécessaire qu'elles évoluent vers un choix personnel de s'engager.

À l'instar des motivations, le choix de s'engager dans le groupe n'est pas statique et doit être renouvelé au cours de la démarche, particulièrement dans des périodes plus difficiles. Plusieurs moyens visant à maintenir et à renouveler le choix d'engagement ont d'ailleurs été identifiés dans les propos des participants. Ces moyens peuvent notamment prendre la forme d'un rappel des objectifs visés (p. ex. offrir un environnement sain à ses enfants, vivre une relation amoureuse saine) ou d'un discours intérieur de motivation et de réflexions, visant à s'encourager en constatant les progrès réalisés ou en s'inspirant des autres membres: « [Quand] une personne commence à changer, ça, pour moi, c'est très motivant. Tu te dis : "Regarde, il l'a fait, j'ai aucune raison pour ne pas le faire." » (participant d'un groupe de discussion, 8 rencontres) 


\title{
Prendre le risque de s'investir dans la démarche
}

Le choix de s'engager se traduit entre autres par la participation au groupe. Pour plusieurs participants, une stratégie consiste à prendre le risque de s'investir dans la démarche, entre autres en prenant la parole et en partageant ses préoccupations avec les autres membres du groupe. Elle est parfois teintée par l'évaluation des coûts et avantages associés à l'engagement dans le groupe :

\begin{abstract}
C'est l'inconnu: tu viens ici, tu appréhendes, tu te dis «dans quoi je me suis embarqué ? » Et ce sont tous des étrangers. Je conte ma vie devant du monde que je ne connais pas. Et en même temps, tu te fais poser des questions : « Est-ce que je parle ? Je ne parle pas ? Je fais quoi ? » Et en même temps, tu te rends compte «si je ne parle pas, je ramènerai jamais mes enfants, je ne réglerai jamais mes choses ». À un moment donné, tu te mets à parler. Là, souvent, les intervenants vont te dire : «Ben ça a été le fun. Ce soir, tu as parlé, félicitations. » (...) [Je me dis] : ils ont bien aimé ça et, moi, ça m'a fait du bien. Regarde, on va recommencer l'autre semaine. » (participant 23, 8 rencontres)
\end{abstract}

Cet extrait rejoint le point de vue de plusieurs hommes pour lesquels il est primordial de prendre la parole, dans le groupe et dans les discussions informelles (p. ex. durant la pause), même en dépit de la timidité ou de doutes quant à la pertinence de leurs propos. Plusieurs soulignent aussi l'importance de s'ouvrir aux intervenants ou aux autres membres pour partager les difficultés rencontrées lors de la démarche. Ces moyens étaient d'ailleurs parmi les plus souvent suggérés par les hommes en réponse aux vignettes présentées : s'ouvrir aux autres membres, aux intervenants, à la direction de l'organisme, à sa conjointe ou ses proches. Prendre la parole est donc une stratégie qui inclut un continuum d'engagement dans le groupe, allant de la participation aux échanges à la révélation de soi en cas de difficultés. Ces stratégies du choix et de la prise de parole suggèrent qu'une part importante de l'engagement relève de la responsabilité des hommes. Par contre, les expériences vécues en groupe peuvent influer sur l'évaluation constante des coûts et avantages associés à l'engagement et, ultimement, favoriser ou non le renouvellement du choix de s'engager.

\section{Stratégie associée aux intervenants, aux membres et au fonctionnement du groupe}

Plusieurs participants ont en effet évoqué l'accueil, le climat de travail, le fonctionnement du groupe, les actions de l'intervenant et la contribution des autres membres du groupe comme des éléments susceptibles de favoriser leur engagement dans le groupe. Ces éléments sont reliés au fonctionnement du groupe comme microsystème et ont été regroupés sous une même stratégie : l'instauration d'un climat de confiance et de travail.

La création d'un lien de confiance s'amorce dès le premier contact avec l'organisme, qui est d'ailleurs crucial pour favoriser l'engagement. Plusieurs participants ont en effet souligné l'importance d'un accueil chaleureux et du professionnalisme des intervenants dans leur choix de s'engager : «Ce qui m'a influencé à continuer mes démarches, c'est que je n'ai senti aucun préjugé. Au contraire, j'ai senti plus un encouragement. Ça a été tout de suite positif pour moi. C'est ça qui m'a aidé vraiment à persévérer pis à continuer ». (participant 27, 17 rencontres) 
Au sein du groupe, le climat de confiance a été évoqué sous plusieurs angles et, en général, les hommes décrivaient une atmosphère d'aisance, détendue et qui laisse place à l'humour malgré le sérieux du propos: «Les intervenants s'assurent vraiment qu'on est bien dans le groupe, qu'on se sent à notre place et qu'on se sent à notre aise de parler. » (participant 5, 20 rencontres) Ils évoquaient aussi une atmosphère empreinte de respect, de non-jugement, d'ouverture, de soutien et d'écoute. Ces conditions favorisent l'instauration d'un climat de travail propice à l'engagement: « On avait tous hâte de revenir parce qu'on venait chercher de l'énergie, du gaz. Ça aidait à continuer. Et de là l'engagement. » (participant 11, 20 rencontres)

Le fonctionnement du groupe est important pour instaurer un climat de confiance et de travail, notamment par des normes favorables à la participation et à la révélation de soi. La gestion des normes a été identifiée à plusieurs reprises en réponse aux vignettes présentées, dont celle où des membres du groupe se moquent du personnage : «Je crois que les intervenants peuvent [dire] : "Regardez, les gars, ça fait cinq rencontres qu'il fait, il n'est pas encore à l'aise... Pouvezvous l'aider à le mettre à l'aise ?" » (participant 1, 19 rencontres)

Ensemble, les intervenants et les membres, par leurs contributions, construisent le climat de travail du groupe. Outre celles déjà mentionnées, plusieurs hommes ont décrit comment certaines actions concrètes des intervenants ont permis d'instaurer un climat de travail favorable à l'engagement. Interpeller les membres plus discrets, questionner ou confronter, encourager et souligner les progrès réalisés, sensibiliser aux situations à risque de récidive et donner des outils concrets pour éviter la violence figurent au registre de ces interventions.

Le respect et la contribution des autres membres du groupe sont tout aussi essentiels à l'instauration d'un climat de confiance et de travail qui suscite l'engagement. Plusieurs participants ont mentionné que le soutien et le non-jugement des pairs constitue une condition essentielle pour stimuler l'engagement dans le groupe : « L'implication des personnes quand je racontais mes situations et... ne pas me sentir jugé. Les personnes, même, en remettaient par rapport à leur vécu. Ça a vraiment favorisé mon engagement et mon avancement dans ma démarche. Ça m'a permis d'aller chercher des outils. » (participant 22, 20 rencontres)

\section{Stratégies associées aux interactions entre le groupe et les autres sphères de vie}

Plusieurs propos des participants soulignent l'importance des interactions entre les différentes sphères de vie des hommes (p. ex. famille, travail, amis) sur leur engagement dans le groupe. Les stratégies identifiées par les hommes rencontrés concernent le soutien des proches et la gestion d'éléments susceptibles d'inhiber l'engagement dans le groupe.

\section{Recevoir du soutien des proches}

Du point de vue des répondants, le soutien des proches (p. ex. ex-conjointe, conjointe, enfants) notamment sous forme d'encouragements, d'écoute et de présence en cas de difficultés, constitue une stratégie très favorable à l'engagement.

C'est sûr que si tu as une conjointe, ben elle, tous les côtés positifs qu'elle voit, elle devrait le souligner, elle devrait t'encourager, elle devrait tout te faciliter pour que tu 
te rendes jusqu'au bout de tes rencontres. Vraiment, moi, je dis qu'elle devient ton partenaire, ton copilote pis si un soir tu "feeles" pas, elle est là. Elle va te dire : "Ben crime, continue !" (participant 24, 8 rencontres)

Considérant les dynamiques en cause dans le problème de violence conjugale, le soutien des proches, surtout de la conjointe ou de l'ex-conjointe, soulèvent certaines réserves qui seront abordées dans la discussion.

\section{Prioriser la démarche du groupe}

Les participants ont aussi identifié des stratégies afin de composer avec des éléments extérieurs au groupe susceptibles de nuire à leur engagement. Ces stratégies consistent souvent à prioriser la démarche de groupe par rapport à d'autres sphères de vie. Par exemple, en lien avec le travail, la négociation de remplacements, de récupération de temps et de réorganisation de l'horaire de travail avec l'employeur constituent des moyens que plusieurs hommes rencontrés ont utilisés pour maintenir leur engagement. Plusieurs ont également suggéré de modifier leur réseau social s'il risque de nuire à l'engagement. Par exemple, en réponse à la vignette où un homme songe à abandonner le groupe en raison du dénigrement de ses amis, plusieurs ont répondu, souvent sans équivoque, que cet homme doit changer son réseau de pairs : " En partant, il n'a pas un bon entourage. Ça fait partie de vouloir s'en sortir, "clairer" ce qui n'est pas bon autour de toi. » (participant 26; 8 rencontres) Ces solutions, en apparence un peu drastiques, reflètent peut-être les limites de l'approche méthodologique utilisée, discutées plus loin.

\section{Stratégies associées aux politiques des organismes}

Plusieurs hommes ont identifié des éléments associés aux politiques des organismes qui dispensent les services susceptibles de favoriser leur engagement dans les groupes. Ces éléments concernent essentiellement le soutien de l'organisme en cas de difficultés (p. ex. la possibilité d'avoir un entretien avec les intervenants en cas de besoin ou de changer de groupe). La stabilité des dyades d'intervenants a aussi été identifiée comme une seconde stratégie contribuant à créer un lien de confiance avec les intervenants et l'organisme : « Je le vois plus comme un niveau de confiance, parce que le groupe, tu viens, tu partages, pis comme notre groupe a une bonne complicité, si demain matin, un des deux [intervenants] partirait, au stade où on est rendu, je pense que c'est clair que ça viendrait nous ralentir (...) me semble que ça donne un coup de mort. » (participant d'un groupe de discussion, 8 rencontres)

\section{Stratégie associée au contexte social}

La seule stratégie liée au contexte social soulevée par les participants a trait à la démystification du problème de la violence conjugale. Quelques hommes suggèrent en effet de faire en sorte que les groupes pour conjoints aux comportements violents ne soient pas associés à l'étiquette de « batteurs de femmes » ou seulement à la violence physique. Cela permettrait selon eux à plus d'hommes de reconnaître leur problème de violence conjugale, et donc de rendre les groupes plus accessibles à ceux qui en ont besoin. Cette plus grande accessibilité favoriserait donc l'engagement initial dans une démarche de changement. 


\section{Discussion}

Les stratégies identifiées dans les propos des hommes rencontrés suggèrent différentes pistes de réflexion en lien avec l'engagement dans les groupes visant à contrer la violence conjugale. Le choix de s'engager, les stratégies qui favorisent la consolidation de ce choix et le réalisme des stratégies évoquées par les participants figurent parmi ces pistes de réflexion.

\section{Le choix de s'engager, une décision au cœur des dimensions de l'engagement}

Les résultats obtenus soulignent le rôle crucial du choix de s'investir dans le processus d'engagement dans les groupes. En effet, l'engagement résulte d'un choix qui doit être fait, puis renouvelé en cours de démarche, particulièrement dans les périodes plus difficiles. Ainsi, ce choix apparaît comme l'élément central qui sous-tend l'adoption des stratégies en vue de s'engager dans une démarche de groupe.

Les stratégies identifiées par les participants rejoignent plusieurs dimensions du modèle d'engagement de Macgowan (2006). Par exemple, le choix d'engagement peut inciter les hommes à trouver des moyens de gérer des conflits d'horaires entre le groupe et le travail (présence), ou à prendre le risque de prendre la parole en groupe (contribution au groupe). Il peut aussi aider les hommes à ignorer des commentaires négatifs sur la démarche et à continuer à s'y investir (travailler sur ses problèmes). Les hommes ont aussi souligné l'importance des moyens favorisant le climat de confiance et de travail, ainsi que la stabilité des dyades d'intervenants, qui peuvent être associées aux dimensions relationnelles de ce modèle. Ces résultats soulignent l'importance d'aborder l'engagement comme un concept aux dimensions variées et sur lesquelles il est possible d'agir favorablement, notamment en soutenant le choix de s'engager.

\section{Des stratégies pour soutenir et consolider le choix de s'engager}

De façon générale, les stratégies identifiées par les hommes rencontrés sont surtout centrées sur eux-mêmes et sur leurs expériences au sein du groupe, ce qui rejoint d'ailleurs l'état des connaissances qui identifient peu d'éléments extérieurs au groupe susceptibles de favoriser l'engagement (Campbell et al., 2010 ; Chovanec, 2014 ; Holdsworth et al., 2014 ; Zalmanowitz et al., 2013). Sur le plan personnel, les hommes ont identifié plusieurs stratégies qu'il est possible de qualifier d'introspectives ( $p$. ex. discours intérieur). Ces stratégies peuvent donc souvent passer inaperçues, voire suggérer une absence d'engagement. Or, ces stratégies ne sont pas automatiquement synonymes de désengagement, particulièrement en début de démarche. Elles peuvent au contraire constituer une étape dans le processus de construction du choix d'engagement au fil des expériences de groupe, notamment par l'évaluation des avantages et des coûts. L'engagement n'en implique pas moins un investissement minimal dans des dimensions que l'on pourrait qualifier de plus actives (p. ex. contribution aux échanges, Macgowan, 2006) et le défi consiste alors à aider les hommes à extérioriser ou à actualiser pleinement leur engagement.

Contrairement à d'autres écrits (Holdsworth et al., 2014 ; Semiatin et al., 2013 ; Ting et al., 2009), les hommes rencontrés n'ont identifié aucune stratégie liée à la réalisation d'activités hors 
groupe. Ce résultat peut s'expliquer par la nature des groupes étudiés. En effet, à la différence des autres recherches, les groupes étudiés n'incluent pas d'entretiens motivationnels ou d'activités hors groupe formellement établies. Ainsi, les intervenants laissent une large place aux échanges entre les membres et utilisent principalement les situations que les hommes apportent au groupe pour construire le travail. Tout en préservant l'orientation moins structurée des programmes étudiés dans la présente recherche, l'inclusion d'un entretien motivationnel et de quelques activités hors groupe pourrait représenter une stratégie intéressante à implanter pour favoriser l'engagement. Ces stratégies pourraient notamment aider les hommes dans le passage de stratégies plus introspectives à des plus actives pour actualiser pleinement leur engagement.

Les résultats soulignent par ailleurs l'importance du rôle des intervenants afin de soutenir le choix d'engagement, notamment par l'accueil et l'instauration d'un climat de confiance et de travail. Par exemple, les résultats obtenus rejoignent ceux de Chovanec $(2012 ; 2014)$ et suggèrent d'utiliser différents moyens visant à renforcer les relations entre les membres du groupe. Ces relations teintent le climat du groupe et font partie des expériences qui appuient ou font obstacle au choix de s'engager dans la démarche. Cet aspect du groupe semble particulièrement important dans les groupes orientés sur le processus où, en comparaison avec les programmes psychoéducationnels, le fonctionnement repose davantage sur la révélation de soi des membres. De concert avec d'autres recherches (Chovanec, 2012 ; 2014 ; Holdsworth et al., 2014), les résultats permettent aussi de dégager divers moyens par lesquels les intervenants peuvent aider les hommes à s'engager graduellement dans le groupe : interpeller des membres plus silencieux, veiller au bon fonctionnement du groupe, encourager la prise de risque et souligner les progrès réalisés. Les intervenants peuvent aussi renforcer positivement certaines initiatives hors groupe, notamment celles liées à la priorisation de la démarche de groupe par rapport à certains éléments susceptibles de freiner leur engagement (p. ex. horaire de travail).

Enfin, bien que les hommes aient mentionné peu de stratégies extérieures au groupe, il nous semble important de continuer d'explorer, autant sur le plan de la recherche que des pratiques, comment les politiques des organismes et le contexte social entourant la violence conjugale peuvent influer sur le choix des hommes de s'engager dans une démarche de groupe. Ces éléments illustrent en effet le processus de construction de ce choix, qui peut débuter en amont même de l'intégration au groupe, par exemple par la démystification du problème de violence conjugale.

\section{Des stratégies réalistes?}

Les résultats de cette étude suggèrent que les proches, surtout la conjointe, l'ex-conjointe, et les enfants, peuvent renforcer ou inhiber l'engagement. Bien que certains aient tenu des propos nuancés, les attentes de plusieurs hommes à l'égard du soutien que leur accorde, ou devrait leur accorder leur conjointe, doivent être considérées avec circonspection. Étant donné les efforts qu'ils investissent dans leur démarche, certains participants semblent se représenter le soutien de leur conjointe comme un dû. Or, si le soutien des proches peut favoriser l'engagement, ces proches sont généralement les victimes de la violence exercée par l'homme. Entretenir des attentes envers les victimes peut augmenter les risques de blâme à leur endroit si elles n'offrent 
pas ce soutien, blâme qui peut mener aux violences psychologiques. Il importe donc de remettre aux hommes la responsabilité de leur engagement, de modérer leurs attentes à l'égard du soutien de leurs proches et d'être vigilant quant aux risques de violence.

\section{Limites}

Les stratégies identifiées dans cette étude sont celles d'hommes qui s'estimaient assez engagés et on peut penser qu'un plus grand nombre d'hommes ayant abandonné leur démarche aurait amené un éclairage différent. De même, lors des entrevues, les hommes étaient invités à se prononcer sur des vignettes afin d'identifier des stratégies favorisant l'engagement dans les groupes. En réponse à ces vignettes, les hommes ont été prompts à suggérer des stratégies, peut-être bénéfiques mais un peu expéditives ( $p$. ex. changer son réseau de pairs), avec peu d'égards quant à l'ampleur des efforts qu'elles demandent. D'ailleurs, des 15 participants ayant suggéré que le personnage de la vignette change son réseau social, un seul mentionne l'avoir fait. Plusieurs hommes ont néanmoins souligné avoir eu à composer avec des commentaires négatifs de leur entourage au cours de leur démarche. La grande majorité d'entre eux a préféré ignorer ces commentaires plutôt que de modifier leur réseau. Cela laisse supposer un écart entre les stratégies proposées pour résoudre des situations fictives et celles qui sont plus réalistement applicables dans la réalité. Ainsi, si l'utilisation de vignettes s'est avérée intéressante pour stimuler les réflexions des hommes pendant les entrevues, sur le plan méthodologique, cette stratégie ne saurait être utilisée seule dans le cadre d'une autre recherche sur le même thème. Une autre limite a trait à l'homogénéité ethnoculturelle des participants et on peut penser que des hommes de communautés culturelles, possiblement confrontés à des obstacles particuliers quant à leur engagement (p. ex. langue), auraient pu mentionner d'autres stratégies.

\section{Conclusion}

Cet article s'intéressait aux stratégies qui favorisent l'engagement des hommes dans les groupes visant à contrer la violence conjugale. Les résultats suggèrent que la décision de s'engager constitue une dimension centrale du processus d'engagement. La majorité des stratégies identifiées par les hommes dépasse la présence au groupe et s'adresse aux différentes dimensions du modèle de l'engagement dans les groupes de Macgowan (2006). Le rôle des intervenants consiste dès lors à soutenir le choix d'engagement des hommes, notamment en renforçant les relations entre les membres du groupe, en encourageant la prise de risques et en soulignant les progrès réalisés. Les résultats suggèrent aussi de remettre aux hommes la responsabilité de leur engagement et de tempérer leurs attentes à l'égard de leur environnement. Les propos des participants à cette recherche laissent croire que le choix d'engagement agit comme facteur de protection contre les abandons. Considérant que la majorité des participants s'estimaient engagés, il serait intéressant d'interroger des hommes qui n'ont pas encore pris cette décision afin d'approfondir notre compréhension du processus qui mène à ce choix. 
ROY, Valérie Roy

Ph.D., T.S., professeure agrégée à l'École de service social de l’Université Laval.

LABARRE, Michel

Étudiant au doctorat en service social à l’Université Laval

DROUIN, Marie-Ève

professionnelle de recherche

Centre de recherche sur l'adaptation des jeunes et des familles à risque (JEFAR)

\section{RÉFÉRENCES}

Bennett, L.W., C. Stoops, C. Call et H. Flett (2007). «Program completion and re-arrest in a batterer intervention system », Research on Social Work Practice, vol. 17, no 1, p. 42-54.

Bowen, E. (2011). The rehabilitation of partner-violent men, Malden (MA), Wiley-Blackwell.

Brodeur, N., G. Rondeau, S. Brochu, J. Lindsay et J. Phelps (2008). « Does the transtheoretical model predict attrition in domestic violence treatment programs? », Violence and Victims, vol. 23, no 4, p. 493-507.

Bronfenbrenner, U. (1986). «Ecology of the family as a context for human development: Research perspective », Developmental Psychology, vol. 22, no 6, p. 723-742.

Campbell, M., J.A. Neil, P.G. Jaffe et T. Kelly (2010). «Engaging abusive men in seeking community intervention: A critical research \& pratice priority », Journal of Family Violence, vol. 25, no 4, p. 413-422.

Chovanec, M.G. (2012). «Examining engagement of men in a domestic abuse program from three perspectives: An exploratory multi-method study », Social Work With Groups, vol. 35, no 4 , p. 362-378.

Chovanec, M.G. (2014). The power of learning and men's stories in engaging abusive men in the changing process: Qualitative study across programs. Social Work with Groups, vol. 37, no 4, p. 331-347.

Contrino, K.M., K.H. Dermen, T.H. Nochajski, W.F. Wieczorek et P.K. Navratil (2007). «Compliance and learning in an intervention program for partner-violent men », Journal of Interpersonal Violence, vol. 22, no 12, p. 1555-1566.

Crane, C. A., et C.I. Eckhardt (2013). «Evaluation of a single-session brief motivational enhancement intervention for partner abusive men », Journal of Counseling Psychology, vol. 60 , no 2, p. 180-187.

Gouvernement du Québec (1995). Politique d'intervention en matière de violence conjugale. Prévenir. Dépister. Contrer la violence conjugale. Québec, Gouvernement du Québec. 
Holdsworth, E., E. Bowen, S. Brown et D. Howat (2014). «Offender engagement in group programs and associations with offender characteristics and treatment factors: A review », Aggression and Violent Behavior, vol. 19, no 2, p. 102-121.

Jewell, L.M., et J.S. Wormith (2010). « Variables associated with attrition from domestic violence treatment programs targeting male batterers: A meta-analysis », Criminal Justice and Behavior, vol. 37, no 10, p. 1086-1113.

Macgowan, M.J. (2006). The group engagement measure: A review of its conceptual and empirical properties. Journal of Groups in Addiction and Recovery, vol. 1, no 2, p. 33-52.

Miles, M.B., et A.M. Huberman (2003). Analyse de données qualitatives (2e éd.), Paris, De Boeck Université.

Ministère de la Sécurité publique (2015). Criminalité dans un contexte conjugal au Québec. Faits saillants 2013, Montréal, Direction de la prévention et de l'organisation policière.

Murphy, C.M., E.L. Linehan, J.C. Reyner, P.H. Musser et C.T. Taft (2012). « Moderators of response to motivational interviewing for partner-violent men », Journal of Family Violence, vol. 27 , no 7 , p. 671-680.

Organisation mondiale de la Santé (2014). Violence d'un partenaire intime et violence sexuelle à l'encontre des femmes, http://www.who.int/mediacentre/factsheets/fs239/fr/ (consulté le 9 mars 2015).

Patton, M.Q. (2002). Qualitative research and evaluation methods (3e éd.). Thousand Oaks (CA), Sage.

Rondeau, G., S. Brochu et G. Lemire et N. Brodeur (1999). La persévérance des conjoints violents dans les programmes de traitement qui leur sont proposés, Montréal, Centre de recherche interdisciplinaire sur la violence familiale et la violence faite aux femmes.

Scott, K., C. King, H. McGinn et N. Hosseini (2011). « Effects of motivational enhancement on immediate outcomes of batterer intervention », Journal of Family Violence, vol. 26, no 2, p. 139-149.

Semiatin, J.N., C.M. Murphy et J.D. Elliott (2013). « Observed behavior during group treatment for partner-violent men: Acceptance of responsibility and promotion of change », Psychology of Violence, vol. 3, no 2, p. 126-139.

Statistique Canada (2015). La violence familiale au Canada : Un profil statistique, 2013. [Ottawa], Centre canadien de la statistique juridique.

Sullivan, R., et J. Claes (2015). « A Different Kind of Fraternity: Psychological Change and Group Dynamics of Male Batterers », Smith College Studies in Social Work, vol. 85, no 1, p. 30-53.

Ting, L., L. Jordan-Green, C.M. Murphy et S.C. Pitts (2009). « Substance use problems, treatment engagement, and outcomes in partner violent men », Research on Social Work Practice, vol. 19 , no 4 , p. 395-406. 
Zalmanowitz, S.J., R. Babins-Wagner, S. Rodger, B.A. Corbett et A. Leschied (2013). «Association of readiness to change and motivational interviewing with treatment outcomes in males involved in domestic violence groups », Journal of Interpersonal Violence, vol. 28, no 5 , p. 956-974. 